\title{
Worm-like robotic systems: Generation, analysis and shift of gaits using adaptive control
}

\author{
Silvan Schwebke ${ }^{1}$, Carsten Behn² \\ 1. Department of Mechatronics, IImenau University of Technology, Germany. 2. Department of Technical Mechanics, \\ University of Technology, Germany.
}

Correspondence: Assistant Professor Carsten Behn. Address: Department of Technical Mechanics, IImenau University of Technology, Max-Planck-Ring 12 (Building F), 98693 IImenau, Germany. Telephone: 49-3677-69-1813. Fax:

49-3677-691-823. Email: carsten.behn@tu-ilmenau.de

Received: J une 30, 2012

Accepted: September 27, 2011

DOI : 10.5430/air.v2n1p12

URL: http://dx.doi.org/10.5430/air.v2n1p12

\section{Abstract}

The starting point of this work is a biologically inspired model of a worm-like locomotion system (WLLS). The mechanical model comprises discrete mass points connected by viscoelastic force actuators. Ground contact is constituted by ideal spikes which act as constraint forces, preventing backward motion for each mass point equipped with them. The distances between each two consecutive mass points are changed by an adaptive controller in order to track a reference trajectory. In combination with the ground contact via spikes, this results in a (undulatory) locomotion of the system.

After presenting the aforementioned model and the adaptive controller, the construction of specific reference functions, which result in certain gaits, is described. For this purpose an existing algorithm is used; it allows for defining the number and succession of the active spikes as well as the resulting velocity. In the following gait examination, simulations for worm systems with four mass points are carried out to find a selection of those gaits most suitable in terms of actuator and spikes load. Prior to implementing the automatic gait change, simulations are carried out to determine the criteria for shifting: actuator and spike forces. With those criteria, the choice of the optimal gait depends on both locomotion speed and ground inclination. An approximation of the forces mentioned before enables a formulation of inclination-dependent speed intervals. This leads to a combination of speed adjustment and gait change that enables optimal crawling for predefined limits of actuator or spike forces.

\section{Key words}

Worm-like locomotion, Spikes, Gait generation, Gait shift, Adaptive control, Uncertain system

\section{Introduction}

Inspired by the biological paradigm earthworm, this paper investigates similar mechanical systems-worm-like locomotion systems (WLLS). Their mechanical model comprises of a chain of interconnected, discrete mass points. These shall be used to mimic the wave of muscle contraction that travels through the earthworm's body and results in locomotion. Therefore, actuators are assumed to be positioned between each two adjacent mass points. This type of 
generation of global movement can be described as a form of undulatory locomotion. According to Ostrowski et al. ${ }^{[9]}$, "Undulatory locomotion is the process of generating net displacements of a robotic mechanism via a coupling of internal deformations to an interaction between the robot and its environment." Akin to the earthworm, the mechanical model has spikes that serve to fix individual segments on the ground.

Regarding the movement pattern of WLLS, current literature ${ }^{[5,6,11,12]}$ often takes the basic idea from the movement of biological worms. However, the mathematical description of these patterns is based on a purely kinematic view, at times just using two states for the system's actuating elements - elongated and contracted. The generation of specific gaits is discussed, but they are not analyzed and compared in terms of dynamics. Such analyzes are the focus of this work. For that, existing theory for gait generation ${ }^{[13]}$ is used; the gaits the authors derive from kinematic and dynamic considerations are now investigated, in simulations of the dynamics of the WLLS. Since all system parameters are assumed to be unknown or, more precisely, uncertain, an adaptive controller is used to achieve the tracking of certain gaits. It does not require the knowledge of system parameters and is able to achieve the control goal via high-gain stabilization.

After the inspection of individual gaits, gait shifting is investigated, with the goal of automatic gait shifting based on the measurement of certain system variables to choose optimal locomotion patterns for given restrictions of, e.g., actuator forces.

\section{Nomenclature}

$a \quad$ number of active spikes for a specific gait, $a=\operatorname{card}(\boldsymbol{A})$

$\boldsymbol{A} \quad$ set of the active spikes' indices

$c_{j} \quad$ spring rates

$d_{j} \quad$ damping constants

$\boldsymbol{e} \quad$ error vector of the distances $\boldsymbol{l}$ between adjacent mass points: $\boldsymbol{e}=\boldsymbol{l}-\boldsymbol{l}_{\text {ref }}$

$e_{j} \quad$ components of the error vector

$f \quad$ frequency of a periodic gait

$g \quad$ absolute value of earth's gravitational acceleration, $g=9.806 \mathrm{~ms}^{2}$

$i \quad$ index, $i=0, \ldots, n$, e.g., for the worm model's mass points

$j \quad$ index, $j=1, \ldots, n$, e.g., for the worm model's spring rates

$k \quad$ index, $k=1, \ldots, n-1$

$k($.$) \quad (time variant) gain of the controller$

l vector of the distances between consecutive mass points

$l_{j} \quad$ distance between mass points $m_{j-1}$ and $m_{j}$

$m_{i} \quad$ mass of segment $i$ of the worm model

$n \quad$ number of outputs (segment distances) of the worm model

$N \quad$ number of mass points of the worm model, $N=n+1$

$P \quad$ power

$T \quad$ period time, $T=f^{-1}$

$u_{j} \quad$ actuating variables

$x_{i} \quad x$-position of the mass point $m_{i}$

$\alpha \quad$ ground inclination in movement direction of the worm model

$\gamma \quad$ factor for gain increase as defined in the adaption law

$\varepsilon \quad$ amplitude of the length variation of the distances $l_{j}$ for a certain gait

$\kappa \quad$ weight factor of the controller's derivative element

$\lambda$ tolerance of the output as defined in the adaption law

$\sigma \quad$ Factor for gain decrease as defined in the adaptation law 


\section{Preliminaries - Worm model and adaptive control}

Starting point is the chain of mass points shown in Figure 1. This chain has one length dimension: $x$. The variables $x_{i}(t)$ $(i=0, \ldots, n)$ are the current coordinates of the segments. The distance between $m_{i}$ and $m_{i-1}$ shall be named $l_{j}(t)=$ $x_{i-1}(t)-x_{i}(t)$. Each mass point is equipped with spikes, which represent the ground contact. They are assumed to be ideal spikes, i.e., they inhibit any backward movement of a mass point. The modeling of this WLLS is described in ${ }^{[14]}$.

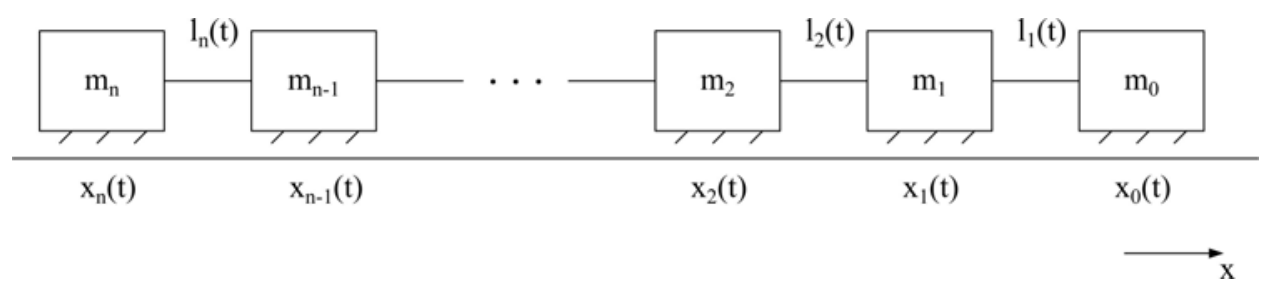

Figure 1. The chain of mass points equipped with spikes.

To achieve the desired movement, inspired by that of the earthworm, the distances $l_{j}(t)$ have to be changed adequately. In this model, actuators are used to apply forces between mass points.

\subsection{Mechanical and mathematical model}

As shown in Figure 2, the WLLS consists of $N=n+1$ mass points, which are linked by $n$ visco-elastic force actuators.

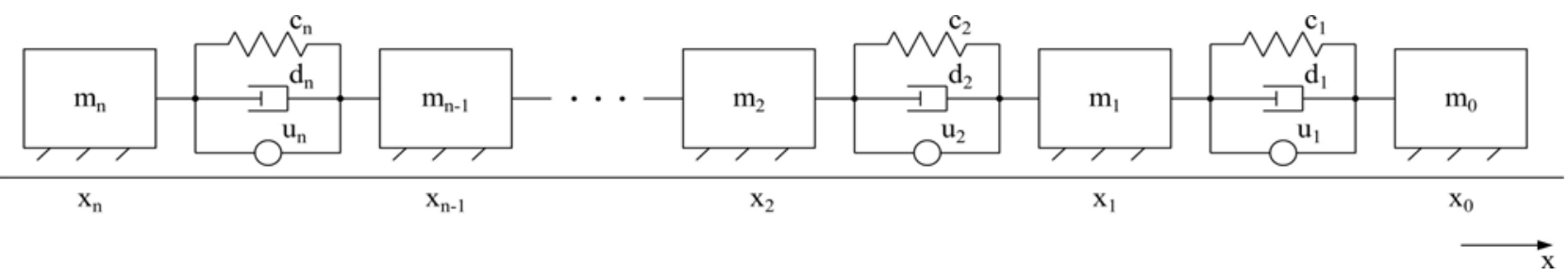

Figure 2. Mechanical model.

In order to obtain a mathematical model, Newton's second law is applied to each mass point, with the forces it is subject to. Figure 3 displays mass point mi in position $x_{i}$ with all forces acting upon it.

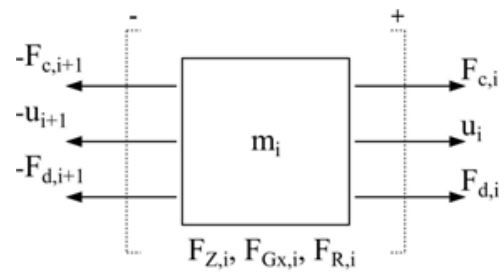

$x_{\mathrm{i}}$

Figure 3. A single mass point $m_{i}$ of the WLLS with all external forces.

The acting forces are $(j=1, \ldots, n)$ :

- Spring forces $F_{c, j}=c_{j}\left(x_{j-1}-x_{j}-l_{0, j}\right)$ and $F_{c, 0}=F_{c, n+1}=0$, where $l_{0, j}$ is the initial length of the spring; 
- $\quad$ Damping forces $F_{d, j}=d_{j}\left(\dot{x}_{j-1}-\dot{x}_{j}\right)$ and $F_{d, 0}=F_{d, n+1}=0$;

- $\quad$ Actuator forces $u_{j}$ and $u_{0}=u_{n+1}=0$;

- $\quad$ Spike forces $F_{Z, i}$, used to model the ideal spikes, therefore fulfilling three conditions ${ }^{[4]}$ :

$$
\dot{x}_{i} \geq 0, F_{Z, i} \geq 0, \dot{x}_{i} F_{Z, i}=0, i=0, \ldots, n .
$$

This means that - exclusively - either the spike force or the velocity of a mass point can be greater than zero.

The spike forces can be considered a special case of static friction forces which act unidirectional and can reach arbitrary non-negative values.

To obtain the mathematical model, the forces have to be expressed explicitly. As reaction forces, they are supposed to compensate all other forces in the case of $\dot{x}_{i}=0$, so that the sum of all forces acting upon $m_{i}$ is zero. These conditions are fulfilled by the following explicit equation given ${ }^{[4]}$ :

$$
F_{Z, i}\left(\dot{x}_{i}, F_{i}\right)=-\frac{1}{2}\left(1-\operatorname{sign}\left(\dot{x}_{i}\right)\right)\left(1-\operatorname{sign}\left(F_{i}\right)\right) F_{i}, i=0, \ldots, n,
$$

Where $F_{i}$ is the sum of all other forces acting upon $m_{i}$.

- The weight of a mass point $F_{G x, i}$ is considered in $x$-direction only and thus depends on the ground inclination $\alpha$. It is derived as

$$
F_{G x, i}(\alpha)=-m_{i} g \sin (\alpha)
$$

- The friction forces $F_{R, i}$ allow for modeling of, e.g., friction according to Stokes' law.

With all of these forces, the coupled differential equations for movement of the mass points can be formulated. They constitute a system of a degree of freedom of $N$ :

$$
\begin{aligned}
m_{0} \ddot{x}_{0}= & -c_{1}\left(x_{0}-x_{1}-l_{0,1}\right)-d_{1}\left(\dot{x}_{0}-\dot{x}_{1}\right)-u_{1}+F_{Z, 0}+F_{G x, 0}+F_{R, 0} \\
m_{j} \ddot{x}_{j}= & -c_{j+1}\left(x_{j}-x_{j+1}-l_{0, j+1}\right)+c_{j}\left(x_{j-1}-x_{j}-l_{0, j}\right)-d_{j+1}\left(\dot{x}_{j}-\dot{x}_{j+1}\right)+d_{j}\left(\dot{x}_{j-1}-\dot{x}_{j}\right) \\
& -u_{j-1}+u_{j}+F_{Z, j}+F_{G x, j}+F_{R, j} \\
m_{n} \ddot{x}_{n}= & -c_{n}\left(x_{n-1}-x_{n}-l_{0, n}\right)+d_{n}\left(\dot{x}_{n-1}-\dot{x}_{n}\right)+u_{n}+F_{Z, n}+F_{G x, n}+F_{R, n}
\end{aligned}
$$

with $j=1, \ldots, n-1$. This system can be influenced using the actuator forces $u_{1}(t)$ through $u_{n}(t)$. They shall serve as inputs to control the distances between mass points in order to follow time-variant functions. With this and the eect of the spike forces, global movement should be achieved.

\subsection{Control}

For global movement, the distances between the mass points should follow prescribed reference trajectories. The variables to be considered are:

- $\quad l_{j}(t)=x_{j-1}(t)-x_{j}(t)$, the distances between adjacent mass points, which represent the system outputs;

- $\quad l_{r e f, j}(t)$, the reference functions of these distances;

- $e_{j}(t):=l_{j}(t)-l_{r e f, j}(t)$, the error of the output variables. 
The task of the controller is to find appropriate actuator forces, depending on the error variables: $\boldsymbol{e} \mapsto \boldsymbol{u}$.

The controller is required to stabilize WLLS with unknown or uncertain (known within an interval) parameters. Therefore, adaptive control ${ }^{[1]}$ is used. The controller contains regular PD-control and a gain adaption law which adjusts the gain of the P- and D-elements in order to achieve stability. The control goal is to track a reference function of the output variables within a given tolerance $\lambda$. This type of $\lambda$-tracking with an adaptive controller has been described ${ }^{[7]}$; the following controller was proposed ${ }^{[3]}$ and examined ${ }^{[8]}$ :

$$
\begin{aligned}
& \boldsymbol{e}(t):=\boldsymbol{l}(t)-\boldsymbol{l}_{\text {ref }}(t) \\
& \boldsymbol{u}(t)=k(t) \boldsymbol{e}(t)+\kappa k(t) \dot{\boldsymbol{e}}(t)=k(t)(\boldsymbol{e}(t)+\kappa \dot{\boldsymbol{e}}(t)) \\
& \dot{k}(t)=\left\{\begin{array}{cc}
\gamma(\|e(t)\|-\lambda)^{2}, & \|e(t)\| \geq \lambda, \\
\gamma(\|e(t)\|-\lambda)^{0.5}, & \lambda+1>\|(e(t))\| \geq \lambda, \\
0, & (\|e(t)\|<\lambda) \wedge\left(t-t_{E}<t_{d}\right), \\
-\sigma k(t), & (\|e(t)\|<\lambda) \wedge\left(t-t_{E} \geq t_{d}\right),
\end{array}\right.
\end{aligned}
$$

with $\gamma>0, \kappa>0, \sigma>0, t_{d}>0, \lambda>0, k(0)=k_{0}>0$.

The adaption law leads to an increase of the gain $k($.$) if the error norm exceeds \lambda$. The quadratic increase in case of $\|e(t)\| \geq \lambda+1$ enables a quick rise of the gain for very large error values; to make sure the gain increase is not too small for error norms that are smaller, but exceed $\lambda$, the square-root function is used to determine $k\left(\right.$.). The variable $t_{E}$ denotes the time of the last entrance of the error norm into the $\lambda$ tube. When $\|e(t)\|$ is smaller than $\lambda$, the gain remains at a constant value for a time delay of $t_{d}$ and decreases exponentially afterwards.

\subsection{Simulation example}

With the mathematical model of the WLLS and the adaptive controller at disposal, the one thing left to be determined to be able to carry out simulations are reference trajectories. For all simulations, the system of differential equations is solved via MatLab, version 7.11.0.574 (R2010b), 32-Bit. The multistep method ode15s is employed, which is adequate for stiff problems. The solver was set up with an absolute tolerance of $10^{-8}$ and a relative tolerance of $10^{-5}$. To ensure numerical stability, the sign(.) function in the formulation of the spike forces (2) is approximated by a continuous function, the hyperbolic tangent: $\operatorname{sign}\left(\dot{x}_{i}\right) \approx \tanh \left(10^{4} \dot{x}_{i}\right)$.

First, the reference functions for the distances $l_{j}(t)$ are set as arbitrarily phase-shifted harmonic oscillations:

$$
\begin{aligned}
& l_{r e f, 1}(t)=l_{0}+\frac{1}{4} l_{0} \sin (2 \pi f t), \\
& l_{r e f, 2}(t)=l_{0}+\frac{1}{4} l_{0} \sin \left(2 \pi f t+\frac{1}{3} \pi\right) \\
& l_{r e f, 3}(t)=l_{0}+\frac{1}{4} l_{0} \sin \left(2 \pi f t+\frac{2}{3} \pi\right) .
\end{aligned}
$$

The following set of parameters is used:

\begin{tabular}{|lll|}
\hline$t \in[0,10 \mathrm{~s}]$ & $l_{0}=1 \mathrm{~m}$ & $\sigma=0.2 \frac{1}{\mathrm{~s}}$ \\
$\alpha=25^{\circ}$ & $f=0.5 \mathrm{~Hz}$ & $t_{d}=2 \mathrm{~s}=f^{-1}$ \\
$m_{i}=1 \mathrm{~kg}$ & $\lambda=0.05 \mathrm{~m}$ & $k_{0}=10 \frac{\mathrm{N}}{\mathrm{m}}$ \\
$c_{j}=10 \frac{\mathrm{N}}{\mathrm{m}}$ & $\gamma=500$ & \\
$d_{j}=5 \frac{\mathrm{kg}}{\mathrm{s}}$ & $\kappa=1 \mathrm{~s}$ & \\
\hline
\end{tabular}


For the error norm in adaption law (5), the Euclidian norm is applied, i. e., $\|e(t)\|=\|e(t)\|_{2}$. Simulation results are shown in Figures 4 and 5.

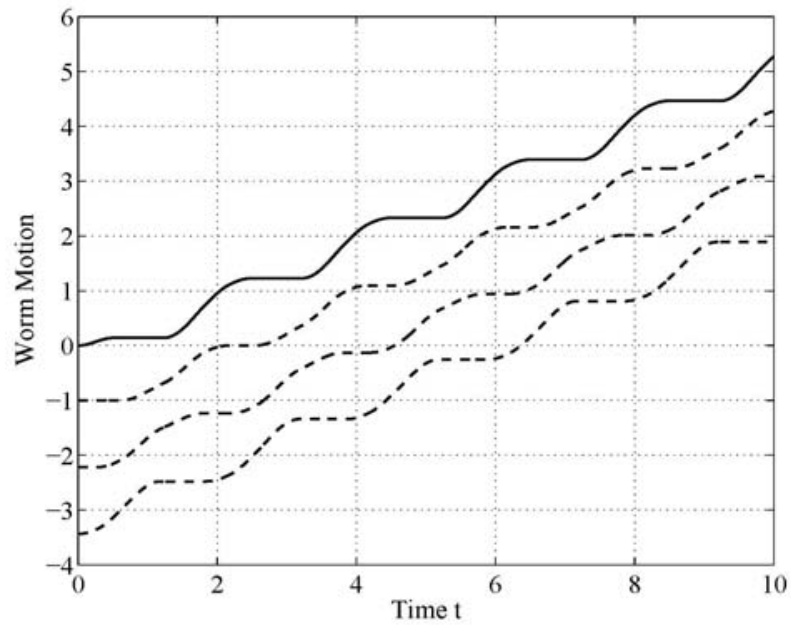

Worm movement vs. time

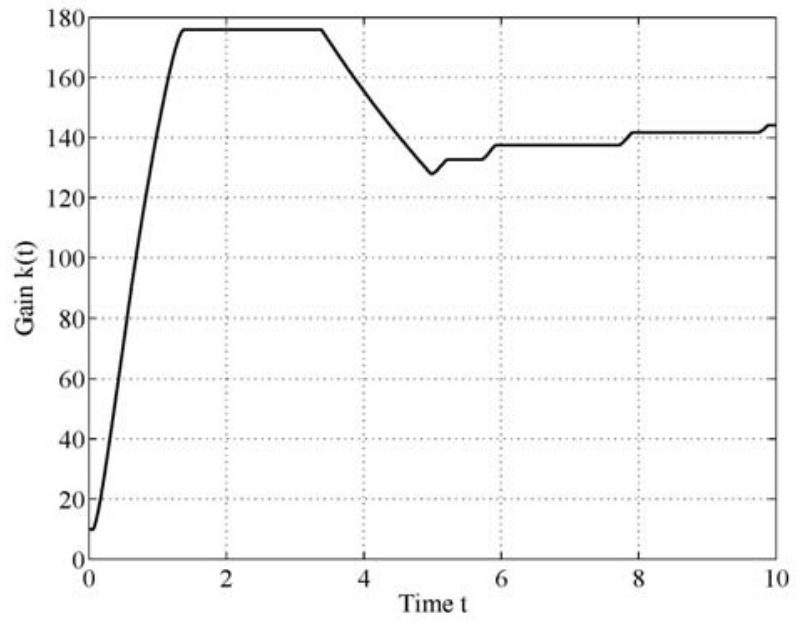

Gain $k(t)$ vs. time

Figure 4. Simulation example, Plots a.
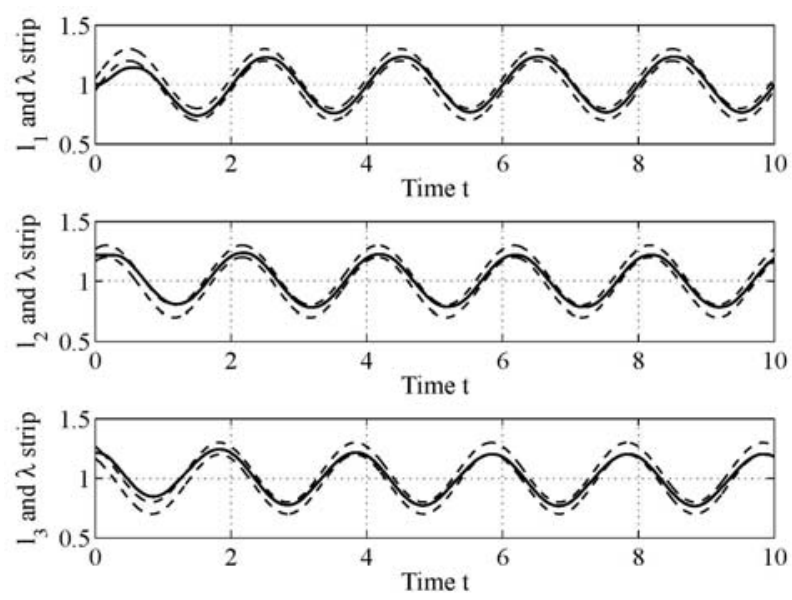

Distances $l_{j}(t)$ and $\lambda$-tube (dashed) vs. time

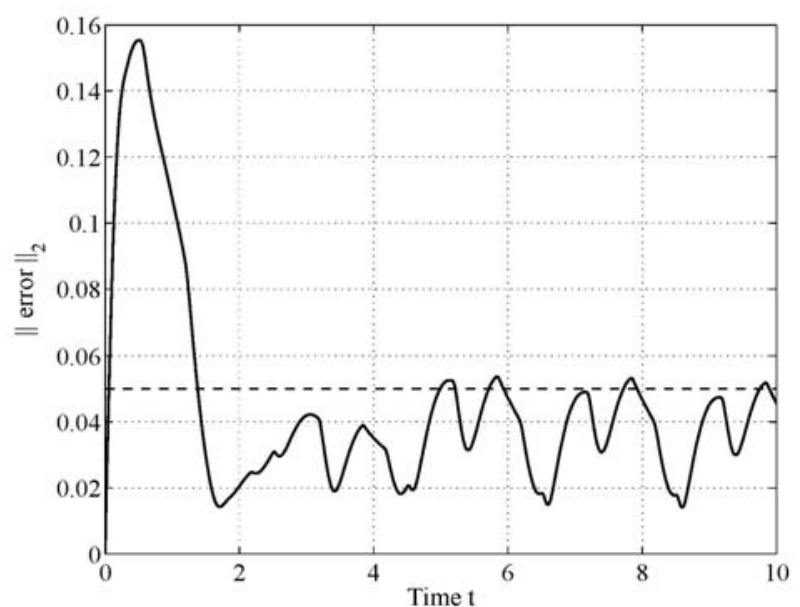

Euclidian norm of the error vector vs. time

Figure 5. Simulation example, Plots b.

Figure 4 (left) displays the positions of the individual mass points vs. time. On the right, the gain is shown. Because of the small initial gain $k(0)$ the error norm (Figure 5 , right) exceeds $\lambda$ considerably, which results in a quick gain increase. Beginning at $t=1.7 \mathrm{~s}$, the error norm is smaller than $\lambda$, the gain is constant and after the delay $t_{d}$, begins to decrease exponentially until the error is too large again. Figure 5 (left) also shows the system outputs - the distances between mass points - which follow the reference functions within a variable error.

The chosen reference functions enable the WLLS to move forward successfully. However, in application one could attempt to find optimal gaits with respect to some goal, e.g., number of resting mass points at every time. Ergo, it is desired to systematically construct reference functions that result in gaits which fulfill detailed requirements regarding the resulting movement. 


\section{Gait analysis}

The construction of reference trajectories $l_{r e f, j}(t)$ that enable the desired global movement of the WLLS consists of two steps ${ }^{[13]}$.

\subsection{Sequence of active spikes}

For every gait, there should be at least one mass point resting at any time, i.e., one or more active spikes. In case this condition is not fulfilled, the speed of all mass points is greater than zero, which means forward skidding of the whole WLLS. First, it is chosen which mass points should have active spikes (i.e. are resting) at which periods of time. The number of active spikes shall be identical for every time and is denoted by $a \in\{1, \ldots, n\}$.

All sequences $\boldsymbol{A}(t)$, which describe the set of active spikes as a function of time, shall be periodic: mode-function.

Remark 3.1: Due to the large number of possible sequences of active spikes, the selection is reduced to those in which the set of active spikes move through the WLLS like a traveling wave. $\boldsymbol{A}(t)$ shall consist of consecutive spike labels. Other, more arbitrary sequences, e. g. " $\{0\} \rightarrow\{3\} \rightarrow\{1\} \rightarrow\{2\}$ " will not be investigated further.

Example 3.2: $n=3, N=4, a=1, A(t)=\{0\} \rightarrow\{1\} \rightarrow\{2\} \rightarrow\{3\}$

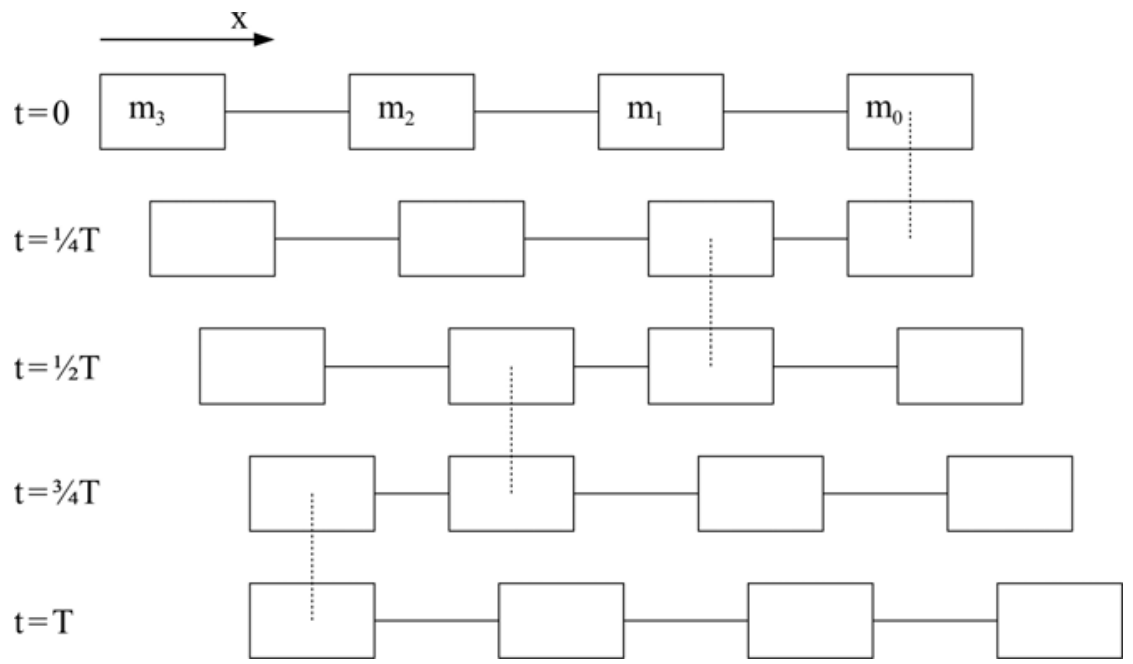

Figure 6. Depiction of one period of the movement pattern with gait a) from Table 1 .

Figure 6 shows an example of a movement pattern in which the active spike travels through the system from head to rear. This is called a gait with the direction left. Unique gaits can be denoted by the initial set of active spikes $\boldsymbol{A}_{0}$ and the direction of the traveling wave of active spikes, $\operatorname{dir}=l$ or $\operatorname{dir}=r$ (Example: Gait $\{0\} \rightarrow\{1\} \rightarrow\{2\} \rightarrow\{3\}$ with $A_{0}=\{0\}$ and $\operatorname{dir}=l$ ("left") - the active spike travels to the left, i.e., from head to rear through the WLLS, as seen in Fig. $6)$. This short notation is used from now on.

Table 1. Spike sequences $\boldsymbol{A}(t)$ for the WLLS with $N=4$, gaits with $a=1$.
a) $\{0\} \rightarrow\{1\} \rightarrow\{2\} \rightarrow\{3\}$
e) $\{0\} \rightarrow\{3\} \rightarrow\{2\} \rightarrow\{1\}$
b) $\{1\} \rightarrow\{2\} \rightarrow\{3\} \rightarrow\{0\}$
f) $\{1\} \rightarrow\{0\} \rightarrow\{3\} \rightarrow\{2\}$
c) $\{2\} \rightarrow\{3\} \rightarrow\{0\} \rightarrow\{1\}$
g) $\{2\} \rightarrow\{1\} \rightarrow\{0\} \rightarrow\{3\}$
d) $\{3\} \rightarrow\{0\} \rightarrow\{1\} \rightarrow\{2\}$
h) $\{3\} \rightarrow\{2\} \rightarrow\{1\} \rightarrow\{0\}$ 
Table 2. Spike sequences $\boldsymbol{A}(t)$ for the WLLS with $N=4$, gaits with $a=2$.
i) $\{0,1\} \rightarrow\{1,2\} \rightarrow\{2,3\} \rightarrow\{3,0\}$
m) $\{0,1\} \rightarrow\{3,0\} \rightarrow\{2,3\} \rightarrow\{1,2\}$
j) $\{1,2\} \rightarrow\{2,3\} \rightarrow\{3,0\} \rightarrow\{0,1\}$
n) $\{3,0\} \rightarrow\{2,3\} \rightarrow\{1,2\} \rightarrow\{0,1\}$
k) $\{2,3\} \rightarrow\{3,0\} \rightarrow\{0,1\} \rightarrow\{1,2\}$
o) $\{2,3\} \rightarrow\{1,2\} \rightarrow\{0,1\} \rightarrow\{3,0\}$
1) $\{3,0\} \rightarrow\{0,1\} \rightarrow\{1,2\} \rightarrow\{2,3\}$
p) $\{1,2\} \rightarrow\{0,1\} \rightarrow\{3,0\} \rightarrow\{2,3\}$

Table 3. Spike sequences $\boldsymbol{A}(t)$ for the WLLS with $N=4$, gaits with $a=3$, dir $=l$.
q) $\{0,1,2\} \rightarrow\{1,2,3\} \rightarrow\{2,3,0\} \rightarrow\{3,0,1\}$
r) $\{1,2,3\} \rightarrow\{2,3,0\} \rightarrow\{3,0,1\} \rightarrow\{0,1,2\}$
s) $\{2,3,0\} \rightarrow\{3,0,1\} \rightarrow\{0,1,2\} \rightarrow\{1,2,3\}$
t) $\{3,0,1\} \rightarrow\{0,1,2\} \rightarrow\{1,2,3\} \rightarrow\{2,3,0\}$

Table 4. Spike sequences $\boldsymbol{A}(t)$ for the WLLS with $N=4$, gaits with $a=3$, dir $=r$.
u) $\{0,1,2\} \rightarrow\{3,0,1\} \rightarrow\{2,3,0\} \rightarrow\{1,2,3\}$
v) $\{3,0,1\} \rightarrow\{2,3,0\} \rightarrow\{1,2,3\} \rightarrow\{0,1,2\}$
w) $\{2,3,0\} \rightarrow\{1,2,3\} \rightarrow\{0,1,2\} \rightarrow\{3,0,1\}$
x) $\{1,2,3\} \rightarrow\{0,1,2\} \rightarrow\{3,0,1\} \rightarrow\{2,3,0\}$

Once the active spikes for specific time intervals, i.e., equal parts of one period, are determined, it can be deduced which $l_{j}(t)$ have to contract, which have to elongate and which have to keep their length in each time interval (see Figure 6 for an example). This algorithm ${ }^{[13]}$ is described in detail. For the following considerations, it is assumed that all mass points without active spikes move forward with the same velocity.

\subsection{Distance reference functions}

In addition to the previous definitions, the detailed $l_{r e f, j}(t)$ functions are yet to be chosen. They should be of class $C^{2}$ (continuous up to the second derivative) to ensure there are no discontinuous steps in the acceleration terms, as this is undesirable in applications. The reference functions are built ${ }^{[13]}$. Starting point $\operatorname{are~}^{2} \sin ^{2}($.)-functions that are used to define parts of the reference signal for time intervals of

$$
t \in\left[p \frac{T}{N},(p+1) \frac{T}{N}\right], p \in \mathbb{N}_{0}
$$

with $\tau=t-p \frac{T}{N}$ as the following functions

$$
\begin{aligned}
& \dot{l}(t)=\varepsilon l_{0} 2 N f \sin ^{2}(\pi f N \tau), \\
& l(t)=l_{0 *}+\varepsilon l_{0} N f \tau-\frac{1}{2 \pi} \varepsilon l_{0} \sin (2 \pi f N \tau) .
\end{aligned}
$$

Here,

- $|\varepsilon| \epsilon[0,1]$ is the relative factor of the maximum contraction/elongation,

- $f$ is the frequency of the $\boldsymbol{A}(t)$-sequence with periodic time $T=\frac{1}{f}$,

- $l_{0}>0$ is the initial (non-contracted, non-elongated) length,

- $l_{0 *}$ is the length at the beginning of a time interval $(\tau=0)$, either $l_{0}$ or $l_{0}(1+\varepsilon)$, depending on the previous movement. 


\subsection{Definitions regarding the analysis of simulations}

For the analysis of all simulations, several variables are evaluated, among them

- Gain,

- Inputs (actuator forces),

- $\quad$ Spike forces

- Mean actuator power,

- Mean velocity,

- $t_{r}$, the time within which the goal of $\lambda$-tracking is reached, i.e., at which $\|e()$.$\| is smaller than \lambda$ and stays smaller for at least one period.

These variables are evaluated for two separate situations,

- The transient interval, which starts at $t=0$ and is often characterized by large gain adjustments,

- The stationary interval, in which the gain stays (roughly) at the same value (Once the outputs stay within the $\lambda$-tube for the duration of $t_{d}$, the gain decreases, until the outputs leave the tube again, which then results in a small gain increase. These small differences are part of the $\lambda$-tracking adaptive controller's characteristics).

The actuator powers $P_{j}$ are given by the product of actuator force and rate of change of its length; their moving average $\bar{P}_{j}$ is:

$$
\bar{P}_{j}(t):=\frac{1}{T} \int_{t-T}^{T} P_{j}(\tau) d \tau, \quad \text { with } \quad P_{j}(t)=u_{j}(t) \dot{l}_{j}(t)
$$

The mean velocity of the center of mass is calculated in the same way:

$$
\bar{v}_{C M}(t):=\frac{1}{T} \int_{t-T}^{T} v_{C M}(\tau) d \tau, \quad \text { with } \quad v_{C M}(t)=\frac{1}{n+1} \sum_{i=0}^{n} \dot{x}_{i} .
$$

The axes units of all plots shown in this paper correspond to the International System of Units (SI).

\subsection{I dentification of optimal gaits}

Using algorithms from ${ }^{[13]}$, piecewise-defined periodic gait reference functions are generated. Now, simulations of the WLLS are carried out in order to evaluate and compare several gaits. In the end, a set of optimal gaits is identified, containing two gaits for every value of $a$ : one with $\operatorname{dir}=l$ and another one with $\operatorname{dir}=r$.

All gaits for the WLLS with $N=4$ that are listed in Example 3.2 are compared. The chosen simulation parameters are given in Table 5. The controller parameters were tested in various simulations and proved to be adequate for a vast span of mechanical system parameters. The adaptive controller (5) is applied. 
Table 5. Parameter set

\begin{tabular}{|lll|}
\hline$t \in[0,20 \mathrm{~s}]$ & $l_{0}=1 \mathrm{~m}$ & $\sigma=0.2 \frac{1}{\mathrm{~s}}$ \\
$\alpha=25^{\circ}$ & $f=0.5 \mathrm{~Hz}$ & $t_{d}=2 \mathrm{~s}=f^{-1}$ \\
$m_{i}=1 \mathrm{~kg}$ & $\lambda=0.05 \mathrm{~m}$ & $k_{0}=10 \frac{\mathrm{N}}{\mathrm{m}}$ \\
$c_{j}=10 \frac{\mathrm{N}}{\mathrm{m}}$ & $\gamma=500$ & $|\varepsilon|=0.4$ \\
$d_{j}=5 \frac{\mathrm{kg}}{\mathrm{s}}$ & $\kappa=1 \mathrm{~s}$ & \\
\hline
\end{tabular}

Complete simulation results can be found ${ }^{[10]}$. Exemplary, some excerpts of these results are shown in Table 6 (transient values) and Table 7 (stationary values). For all variables, the maximum values are evaluated.

Table 6. Gait comparison of the WLLS with $N=4(a=1$, dir $=l)$, transient results

\begin{tabular}{c|cccc|ccccc} 
Gait & $k$ & $\left|u_{1}\right|$ & $\left|u_{2}\right|$ & $\left|u_{3}\right|$ & $F_{Z, 0}$ & $F_{Z, 1}$ & $F_{Z, 2}$ & $F_{Z, 3}$ & $t_{r}$ \\
$\mathbf{A}_{0}$, dir & {$[\mathrm{N} / \mathrm{m}]$} & {$[\mathrm{N}]$} & {$[\mathrm{N}]$} & {$[\mathrm{N}]$} & {$[\mathrm{N}]$} & {$[\mathrm{N}]$} & {$[\mathrm{N}]$} & {$[\mathrm{N}]$} & {$[\mathrm{s}]$} \\
\hline$\{0\}, \ell$ & 305 & 22,7 & 16,1 & 18,9 & 24,2 & 24,3 & 24,2 & 24,3 & 2,49 \\
$\{1\}, \ell$ & 297 & 19 & 17,8 & 19,1 & 24,3 & 26,2 & 24,4 & 24,4 & 2,52 \\
$\{2\}, \ell$ & 307 & 23 & 17,8 & 19,5 & 24,4 & 24,1 & 26,2 & 24,6 & 2,47 \\
$\{3\}, \ell$ & 297 & 23,4 & 16,3 & 22,6 & 24,4 & 24,2 & 24,1 & 24,2 & 2,48
\end{tabular}

Table 7. Gait comparison of the WLLS with $N=4(a=1$, dir $=l)$, stationary results

\begin{tabular}{c|cccc|cccc|cccc} 
Gait & $k$ & $\left|u_{1}\right|$ & $\left|u_{2}\right|$ & $\left|u_{3}\right|$ & $F_{Z, 0}$ & $F_{Z, 1}$ & $F_{Z, 2}$ & $F_{Z, 3}$ & $\bar{P}_{1}$ & $\bar{P}_{2}$ & $\bar{P}_{3}$ & $\bar{v}_{C M}$ \\
$\mathbf{A}_{0}, d i r$ & {$[\mathrm{~N} / \mathrm{m}]$} & {$[\mathrm{N}]$} & {$[\mathrm{N}]$} & {$[\mathrm{N}]$} & {$[\mathrm{N}]$} & {$[\mathrm{N}]$} & {$[\mathrm{N}]$} & {$[\mathrm{N}]$} & {$[\mathrm{W}]$} & {$[\mathrm{W}]$} & {$[\mathrm{W}]$} & {$[\mathrm{s}]$} \\
\hline$\{0\}, \ell$ & 156 & 22,6 & 15,9 & 18,8 & 24,1 & 24,0 & 24,1 & 24,1 & 1,85 & 1,80 & 1,91 & 0,247 \\
$\{1\}, \ell$ & 180 & 19,0 & 16,0 & 18,8 & 24,3 & 24,0 & 24,1 & 24,2 & 1,92 & 1,85 & 1,94 & 0,254 \\
$\{2\}, \ell$ & 186 & 22,8 & 16,7 & 18,8 & 24,3 & 24,0 & 24,0 & 24,2 & 1,94 & 1,86 & 1,95 & 0,255 \\
$\{3\}, \ell$ & 180 & 22,8 & 16,0 & 22,5 & 24,3 & 24,0 & 24,1 & 24,1 & 1,93 & 1,85 & 1,93 & 0,253
\end{tabular}

From this set of gaits with $a=1$ and $\operatorname{dir}=l$, the gait with $\boldsymbol{A}_{0}=\{1\}$ is selected. It requires the smallest maxima of actuating forces. The maximum spike forces are mostly identical to those of other gaits, but once, in the transient case, one of the spikes requires a larger force.

The complete results give a total number of six gaits, two for each $a \in\{1,2,3\}$. This selection of optimal gaits is shown in Table 8.

Table 8. Optimal gaits of the WLLS with $N=4$

\begin{tabular}{|l|l|l|}
\hline$a=1$ & $a=2$ & $a=3$ \\
\hline $\operatorname{dir}=\ell, \mathbf{A}_{0}=\{1\}$ & dir $=\ell, \mathbf{A}_{0}=\{1,2\}$ & $\operatorname{dir}=\ell, \mathbf{A}_{0}=\{2,3,0\}$ \\
$\operatorname{dir}=r, \mathbf{A}_{0}=\{2\}$ & dir $=r, \mathbf{A}_{0}=\{1,2\}$ & $\operatorname{dir}=r, \mathbf{A}_{0}=\{3,0,1\}$ \\
\hline
\end{tabular}

\section{Gait shift}

The change of gaits can help to react to altering environment conditions, such as a varying slope. The following examination consists of an externally triggered gait shift as a first example. Later considerations focus on automatic, internal gait shifting relying solely on measured system variables.

The options for varying the movement pattern are twofold: 
- Changing the number of active spikes (value $a$ ) by switching the $\boldsymbol{A}(t)$ sequence. This is always possible after a full period of the gait sequence has ended because all $l_{j}($.$) have returned to their initial length; it can delay the$ switch by up to one period.

- Changing the gait frequency (value $f$ ) while keeping the general $\boldsymbol{A}(t)$ pattern. This change is possible at any time.

Isolated implementations of either of these principles limit the gait adjustment - especially the mere change of the number of active spikes while keeping a constant frequency, which only allows for $n$ different reference speeds. The reference speed can be obtained from kinematical considerations of the reference functions; it is

$$
\bar{v}_{r e f}(a, f)=(N-a) \varepsilon l_{0} f .
$$

This reference speed can be altered by changing $a$ and $f$. To be able to adjust the speed of the WLLS in an optimal way, a combined switching of the two variables is desired.

Taking the results of Section 3.4, the set of optimal gaits for each number of active spikes $a$ is chosen, for a fixed direction dir. First, these gaits are used to illustrate possible effects of a gait shift; then, propositions for an automatic gait shift are made.

\subsection{Externally triggered gait shift}

To demonstrate advantageous effects of gait shifting, two simulations of the WLLS with $N=4$ are carried out:

- without gait shift: constant gait $a=1, \boldsymbol{A}_{0}=\{1\}$, dir $=l$ with $f=0.5 \mathrm{~Hz}$,

- with gait shift: change from

1. $a=1, A_{0}=\{1\}$, dir $=l$ with $f=0.5 \mathrm{~Hz}$, to

2. $a=2, A_{0}=\{1,2\}$, dir $=l$ with $f=0.4 \mathrm{~Hz}$.

In both cases, the WLLS with $N=4$ crawls through terrain with an increasing slope. For this example, the slope is given as a function of time:

$$
\alpha(t)=\left\{\begin{array}{ll}
25^{\circ} & , t<10 s \\
55^{\circ} & , t \geq 10 s
\end{array} \Rightarrow F_{G x, i}(t)=F_{G x}^{*}(t)= \begin{cases}-m g \sin \left(25^{\circ}\right) & , t<10 s \\
-m g \sin \left(55^{\circ}\right) & , t \geq 10 s\end{cases}\right.
$$

In the first simulation, the initial gait $(a=1)$ is maintained all the way through; for the second simulation, the gait is switched at $t=10 \mathrm{~s}$. This gait shift is implemented by simply swapping the reference functions.

Figures 7,8 and 9 show extracts of the simulations results for comparison.

The worm motion in Figure 7 shows the speed reduction that results from the gait shift (right), whereas the speed is only marginally reduced for the constant gait (left). The larger slope causes the error norm to exceed $\lambda$, so that the gain (see Fig. 8) increases.

With the second gait, $a=2$, the gain reaches higher values. With a constant gait, the spike forces reach significantly higher maximum values (see Figure 9) than before. The gait shift with its reduced locomotion speed helps to avoid the increase of spike force maxima. 


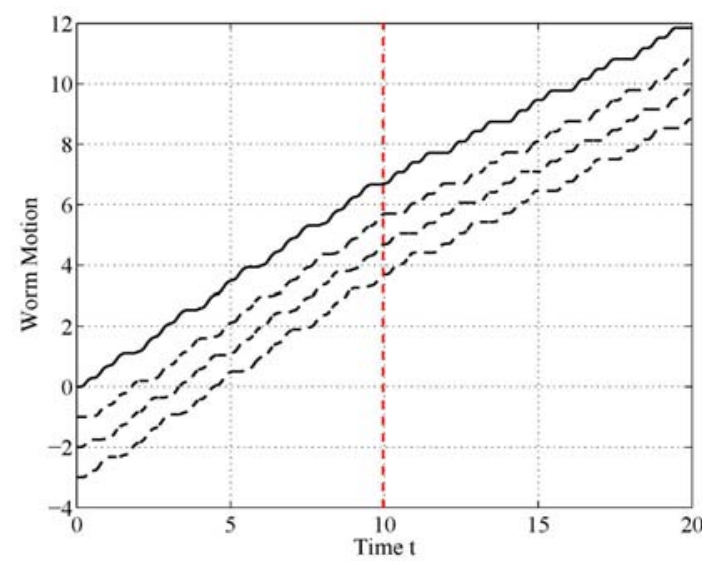

without gait shift

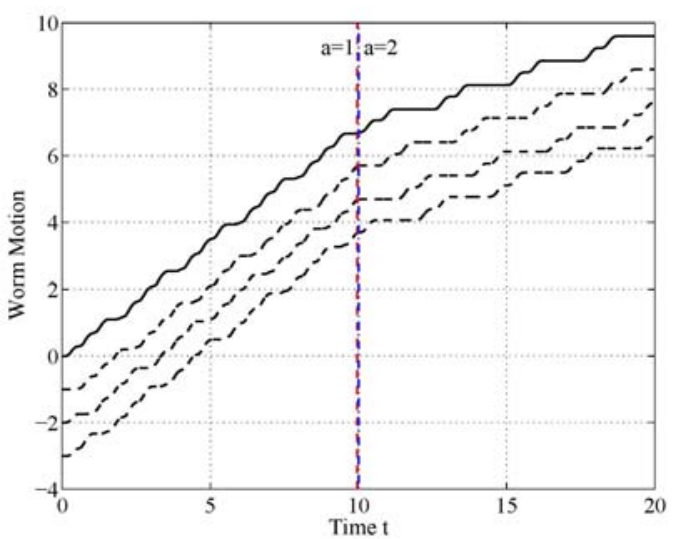

with gait shift

Figure 7. Worm motion for terrain with increasing slope.

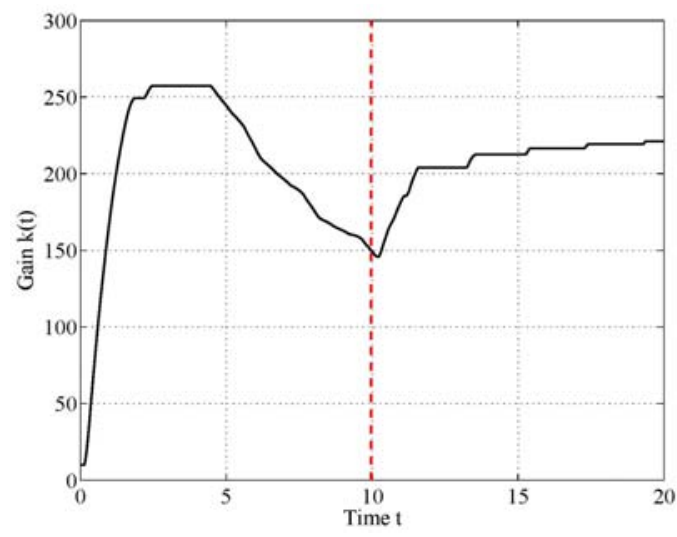

without gait shift

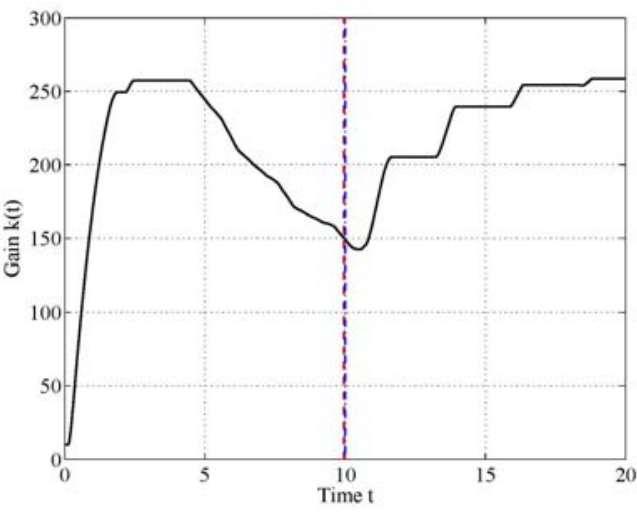

with gait shift

Figure 8. Gain $k(t)$ for terrain with increasing slope.
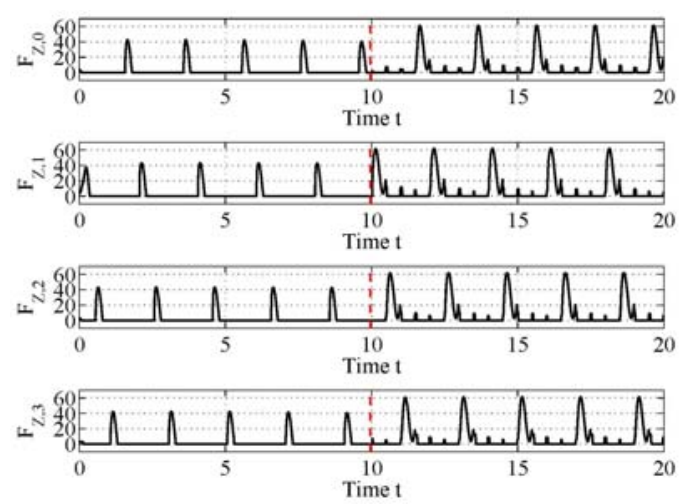

without gait shift
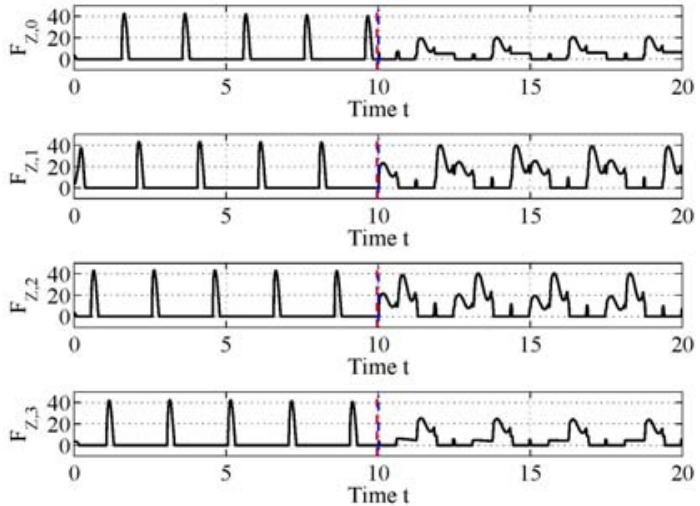

with gait shift

Figure 9. Spike forces for terrain with increasing slope. 
Conclusion 4.1: The gait shift can help to reduce locomotion speed and therefore limit possible increases of spike and actuator forces or actuator powers. The adaptive controller is capable of quickly adjusting the gain to a value that is adequate for different slopes and gaits.

\subsection{Automatic gait shift}

To use different gaits for locomotion in an expedient way, methods shall be found that enable gait shift without external intervention or fixed, time-dependent rules. It is necessary to react to environment conditions such as a certain slope or ground properties. Their influence on known system parameters, e.g., gain or measured actuator forces, should be evaluated in order to choose an adequate gait. A desired attribute of any gait shift rule is a quick reaction to measurements of system variables (e.g., actuator forces) that exceed given limits, as this can lead to adverse effects in applications, such as overstressing of actuators.

\subsection{Criteria for automatic shifting}

Based on Section 4.1, possible criteria for gait shifting can include

- Certain limits of actuator or spike forces, to avoid reaching prohibited values;

- Actuator power, as a maximum or (moving) average value;

- Gain, which is influenced by external conditions such as friction or slope of the terrain;

- Speed (of the center of mass), which can be subject to small changes depending on the slope.

First, a comparison is conducted to analyze the behavior of these criteria when using different gaits, between which a shift is possible. In the following simulations, reference speed $\bar{v}_{r e f}$ and slope $\alpha$ are varied.

\subsubsection{Preliminary investigations: gait analysis}

For the system with $N=4$, gaits with $a=1,2,3$ are compared. The gait frequencies are chosen so that the resulting reference speeds are identical for all $a$. For this, an initial frequency $f_{1}$ is defined; the other frequencies can then be found as per (9):

- $\quad$ For $a=1: f=f_{1}$,

- $\quad$ For $a=2: f=\frac{3}{2} f_{1}$,

- $\quad$ For $a=3: f=3 f_{1}$.

For the simulation, a set of optimal gaits as identified in Section 3.4 is chosen; with the arbitrary direction $d i r=l$, these gaits are

- $a=1, \boldsymbol{A}_{0}=\{1\}$,

- $a=2, \boldsymbol{A}_{0}=\{1,2\}$,

- $\quad a=3, A_{0}=\{2,3,0\}$.

They are compared for a number of different frequencies as well as two different slopes. The parameter set given earlier in Table 5 is used. 
Table 9. Gait analysis $(N=4)$, results for $\alpha=25^{\circ}$

\begin{tabular}{ccc|cc|ccc} 
& & & \multicolumn{2}{|c|}{ transient } & \multicolumn{3}{c}{ stationary } \\
Gait & $f$ & $\bar{v}_{C M}$ & $\max \left(u_{j}\right)$ & $\max \left(F_{Z, i}\right)$ & $\max \left(u_{j}\right)$ & $\max \left(F_{Z, i}\right)$ & $\sum_{\bar{P}_{j}}$ \\
$a$ & {$[\mathrm{~Hz}]$} & {$[\mathrm{m} / \mathrm{s}]$} & {$[\mathrm{N}]$} & {$[\mathrm{N}]$} & {$[\mathrm{N}]$} & {$[\mathrm{N}]$} & {$[\mathrm{W}]$} \\
\hline 1 & 0,1 & 0,098 & 12,0 & 19,3 & 12,0 & 18,0 & 1,22 \\
2 & 0,15 & 0,106 & 12,6 & 18,5 & 12,6 & 18,3 & 1,58 \\
3 & 0,3 & 0,108 & 10,8 & 13,7 & 9,41 & 13,7 & 2,46 \\
\hline 1 & 0,15 & 0,148 & 13,6 & 21,4 & 13,6 & 19,6 & 1,97 \\
2 & 0,225 & 0,161 & 15,1 & 20,7 & 15,1 & 18,2 & 2,67 \\
3 & 0,45 & 0,164 & 14,7 & 18,6 & 12,8 & 17,3 & 4,94 \\
\hline 1 & 0,2 & 0,2 & 16,0 & 23,7 & 16,0 & 21,7 & 2,81 \\
2 & 0,3 & 0,217 & 18,7 & 24,0 & 18,6 & 21,7 & 4,01 \\
3 & 0,6 & 0,222 & 20,8 & 24,5 & 19,6 & 22,3 & 8,18 \\
\hline 1 & 0,25 & 0,251 & 19,1 & 26,3 & 19,0 & 24,3 & 3,74 \\
2 & 0,375 & 0,274 & 23,4 & 28,1 & 23,0 & 25,6 & 5,60 \\
3 & 0,75 & 0,294 & 29,8 & 33,0 & 27,9 & 29,3 & 12,2
\end{tabular}

The results for a slope of $25^{\circ}$ (see Table 9) are:

- $\quad$ The gait with $a=3$ requires the smallest maxima of spike and actuator forces for the smallest displayed speed: $0.1 \frac{\mathrm{m}}{\mathrm{s}}$. For stationary values, this still holds for the speed of $0.15 \frac{\mathrm{m}}{\mathrm{s}}$.

- While the gait with $a=2$ requires almost identical spike force maxima as the gait with $a=1$ for $\bar{v}_{C M} \approx 0.2 \frac{m}{s}$, its actuator force maxima are higher. However, the actual mean velocity of the gait with $a=2$ is almost $10 \%$ larger.

- The gait with $a=1$ is best suited for speed values of about $0.2 \frac{\mathrm{m}}{\mathrm{s}}$ and higher (not shown here) in terms of spike and actuator force maxima.

Table 10. Gait analysis $(N=4)$, results for $\alpha=60^{\circ}$

\begin{tabular}{ccc|cc|ccc} 
& & & \multicolumn{2}{|c|}{ transient } & \multicolumn{3}{c}{$\operatorname{stationary}$} \\
Gait & $f$ & $\bar{v}_{C M}$ & $\max \left(u_{j}\right)$ & $\max \left(F_{Z, i}\right)$ & $\max \left(u_{j}\right)$ & $\max \left(F_{Z, i}\right)$ & $\sum_{P_{j}}$ \\
$a$ & {$[\mathrm{~Hz}]$} & {$[\mathrm{m} / \mathrm{s}]$} & {$[\mathrm{N}]$} & {$[\mathrm{N}]$} & {$[\mathrm{N}]$} & {$[\mathrm{N}]$} & {$[\mathrm{W}]$} \\
\hline 1 & 0,2 & 0,194 & 29,0 & 40,3 & 29 & 39,2 & 4,97 \\
2 & 0,3 & 0,212 & 27,4 & 41,6 & 27,3 & 37,0 & 6,57 \\
3 & 0,6 & 0,215 & 25,2 & 35,0 & 23,9 & 31,3 & 10,4 \\
\hline 1 & 0,25 & 0,242 & 32,2 & 42,5 & 32,1 & 41,9 & 6,41 \\
2 & 0,375 & 0,268 & 32,1 & 45,8 & 31,8 & 40,1 & 8,82 \\
3 & 0,75 & 0,271 & 34,3 & 43,7 & 32,4 & 38,3 & 15,0 \\
\hline 1 & 0,3 & 0,29 & 36,0 & 45,4 & 35,8 & 45,1 & 7,91 \\
2 & 0,45 & 0,325 & 37,6 & 50,8 & 37,2 & 44,2 & 11,3 \\
3 & 0,9 & 0,334 & 45,0 & 53,5 & 42,3 & 47,1 & 20,3 \\
\hline 1 & 0,35 & 0,341 & 40,4 & 49,4 & 39,9 & 48,8 & 9,55 \\
2 & 0,525 & 0,383 & 43,9 & 56,5 & 43,4 & 49,7 & 14,1 \\
3 & 1,05 & 0,415 & 57,5 & 65,4 & 54,7 & 58,2 & 27,3
\end{tabular}

A larger slope of $60^{\circ}$ (Table 10) leads to slightly different results:

- The gait with $a=3$ yields the lowest maxima of spike and actuator forces for speeds of $0.2 \frac{\mathrm{m}}{\mathrm{s}}$ and lower. This still holds for the stationary spike force maxima with the speed of $0.25 \frac{\mathrm{m}}{\mathrm{s}}$; however, the gait with $a=2$ results in lower actuator forces.

- The gait with $a=2$ requires the lowest spike force maxima for $\bar{v}_{C M} \approx 0.3 \frac{\mathrm{m}}{\mathrm{s}}$, whereas its actuator forces are higher than with the gait with $a=1$. However, the gait with $a=2$ reaches a speed that is $10 \%$ higher. 
- In the transient case, the gait with $a=1$ requires the lowest forces for speeds of $0.25 \frac{\mathrm{m}}{\mathrm{s}}$ and higher. For speeds of $0.35 \frac{\mathrm{m}}{\mathrm{s}}$ and higher, it has the lowest spike and actuator forces for the transient and stationary case.

It appears that, with the two criteria of (absolute) actuator force maximum and spike force maximum, there exist speed intervals in which certain gaits are better suited than others. Qualitatively saying, the gait with $a=3$ is optimal for low speeds; in an interval of medium speeds, the gait with $a=2$ requires the smallest forces; for all higher velocities, the gait with $a=1$ is superior.

However, the quantitative speed intervals (i.e., the actual value of thresholds between them) depend on external influences, e.g., the slope, which shifts the speed thresholds, as seen in the results presented above. In an implementation of the automatic gait shift it is not sufficient to start off with $a=1$ and, in case the spike forces exceed a given limit, to switch to $a=2$ or $a=3$ - the spike forces might increase even further. Rather, the gait frequency has to be adjusted within a certain speed interval; and only if the system leaves this speed interval, the number of active spikes, a, should be changed.

In any case, the gait with $a=1$ uses the smallest actuator powers, i.e., requires the least amount of energy for locomotion. Only if there are limits of maximum spike or actuator forces, other gaits should be considered. In the same way, the gait with $a=2$ yields lower actuator powers than $a=3$.

Conclusion 4.2: The gait examinations leads to a few interesting results. If the maximum allowed spike forces or actuator forces are chosen as criteria, an automatic gait shift requires the determination of $n-1$ speed thresholds, at which the number of active spikes should be switched. Between the thresholds, the gait frequency should be adjusted. The exact values of these thresholds can vary during locomotion, as they are not only dependent on internal system parameters, but on external variables such as the slope.

A subsequent problem is the determination of the speed thresholds; it is described in Section 4.4.1. Prior to that, the most important criteria for gait shifting are discussed.

\subsubsection{Actuator forces as a criterion}

The actuator forces directly define the requirements concerning the physical actuators and thus represent an important criterion for gait shift. For further investigation, they are estimated based on reference functions and system parameters.

The reference functions for $\dot{l}_{r e f, j}$ are given in (6), we have

$$
\begin{aligned}
& \dot{l}_{r e f, j}(t)=2 \varepsilon l_{0} f N \sin ^{2}(N \pi f t), \\
& \ddot{l}_{r e f, j}(t)=2 N^{2} \pi f^{2} \varepsilon l_{0} \sin (2 N \pi f t) .
\end{aligned}
$$

With knowledge of $\ddot{l}_{r e f, j}$ and the individual masses, the corresponding inertial forces can be found as per $F=m a$. The expected maximum actuator force depends on the maximum acceleration and the total mass that is accelerated by the actuator. Assuming equal masses $m_{i}=m(i=1 \ldots n)$ the number of masses accelerated by a single actuator shall be called $n_{m}$. The maximum value of this number results from the gait's parameter $a$ :

$$
n_{m, \max }=N-a \text {. }
$$

To find the actuator forces, inertial forces for acceleration and the necessary force to compensate the $x$-component of the masses' weight have to be added, so that the maximum actuator force is given by

$$
\begin{aligned}
u_{\max } & :=\max \left\{\left|u_{j}(t)\right|\right\}=m n_{\max } \max \left\{\left|\ddot{l}_{r e f, j}(t)\right|\right\}+m n_{\text {m, max }} g \sin (\alpha) \\
& =m(N-a)\left[2 N^{2} \pi f^{2} \varepsilon l_{0}+g \sin (\alpha)\right]
\end{aligned}
$$

Spring, damping and friction forces may also influence the amount of the actuator force. They are neglected in this estimation, since they are assumed to be unknown (and not required elsewhere, e.g., to design the controller). Large 
friction and damping might contribute to higher actuator forces, which mean that this is a lower bound estimate.

\subsubsection{Spike forces as a criterion}

In case that the maximum allowed spike forces are known, it could be considered to measure and use them as a shift criterion. This way, the speed can be adjusted in order to limit the spike force maxima to certain boundaries. They can be estimated similarly to the actuator forces; this is presented in Section 4.4.1.

\subsubsection{Other criteria}

Further possibilities of criteria for gait shifting include:

- Actuator powers, which can be measured and be evaluated for gait shifting in order to adhere to a given limit of the maximum or mean power.

- Gain $k($.$) , which is influenced by the slope and the gait that is used. However, it does not directly represent a$ physical variable that may have to be limited and is not investigated further in this paper.

- Locomotion speed, which varies slightly when different slopes are applied. The allowed variation is defined by the $\lambda$-tube. As the slope-dependent deviation from the reference speed is not known in advance, locomotion speed is not used as a direct criterion here.

\subsubsection{Preventing forward skidding}

In case that the speed of the WLLS exceeds a certain value, it begins to skid forward. This means that all mass points slide forward, while none of the spikes are active. This state should be avoided, as it does not allow for direct control of the global movement.

There is a limit of the accelerations $\ddot{l}_{j}$ that marks the point at which skidding will start. If it is exceeded, the inertial forces of the accelerated/decelerated masses reach higher values than the weight of the other masses: In this case, during the state of acceleration of the $N-a$ moving masses in negative $x$-direction, the other a masses are moved forward. This means that the speed of all masses is greater than zero (for a certain time interval) and no spike is active to provide reactive forces. This idea leads to the following formula ${ }^{[13]}$, for the maximum speed without forward skidding:

$$
\bar{v}_{\text {Limit }}=\sqrt{\frac{1}{2 \pi} \varepsilon l_{0} g \sin (\alpha)\left(1-\frac{a}{N}\right)} .
$$

for the case of equal masses $m_{i}=m, i=1, \ldots, n$. With (12), an upper speed limit could be defined, if the slope is measured (all other variables are gait parameters and known in advance). In later simulations, this is implemented as an upper gait frequency limit.

\subsection{Combined adjustment of frequency and number of active spikes}

The frequency $f$ and the number of active spikes $a$ represent two dimensions which can be used to influence the reference speed. Adjustments of the frequency enable arbitrarily small steps or even continuous functions for changing the reference speed (corresponding to stepping on the gas in automotive terms), whereas changing the number of active spikes can be understood as a type of gear shift. Because of $D o F=2$, an additional rule has to be found to decide which combinations of $a$ and $f$ should be used. For this, the results of the gait analysis in Section 4.3.1 are invoked. They lead to the qualitative observation of certain slope-dependent speed thresholds at which the number of active spikes should be changed. 


\subsubsection{Speed thresholds}

If the actuator or spike forces are considered as primary criteria, the choice of the optimal gait depends on locomotion speed (i.e., gait frequency) as well as current slope. Based on the actuator force estimate (11) that describes the maximum (absolute) actuator force as a function of gait, masses and slope, a similar expression for the spike forces can be formulated. In case of $\dot{x}_{i}=0$, the spike force $F_{Z, i}$ equals the sum of all other forces acting upon mass point mi, with opposite sign:

$$
F_{Z, i}\left(\dot{x}_{i}=0\right)=u_{i+1}-u_{i}-F_{G x, i}+F_{c, i+1}-F_{c, i}+F_{d, i+1}-F_{d, i}-F_{R, i}
$$

For the following estimation, all spring, damping and friction forces are neglected. As shown in parameter studies ${ }^{[10]}$, different damping constants have almost no effect on the spike forces anyway, since larger damping is compensated by higher actuator forces. The estimation of the maximum value of $u_{i+1}-u_{i}$ is equal to the $u_{\max }$-term formulated earlier. The spike force estimate is completed by adding the $x$-component of the weight of mass $m_{i}$ :

$$
F_{Z, i, \max }(f, a)=u_{\max }+m_{i} g \sin (\alpha)=m(N-a)\left[2 N^{2} \pi f^{2} \varepsilon l_{0}+g \sin (\alpha)\right]+m g \sin (\alpha),
$$

which makes $F_{Z, i, \max }$ a function of frequency $f$ (or reference speed, see (9)) and number of active spikes $a$.

The estimated spike force maxima are shown in Fig. 10. For the WLLS with $N=4$ and parameters according to Table 5 , the estimation for different numbers of active spikes $a=1,2,3$ is plotted.

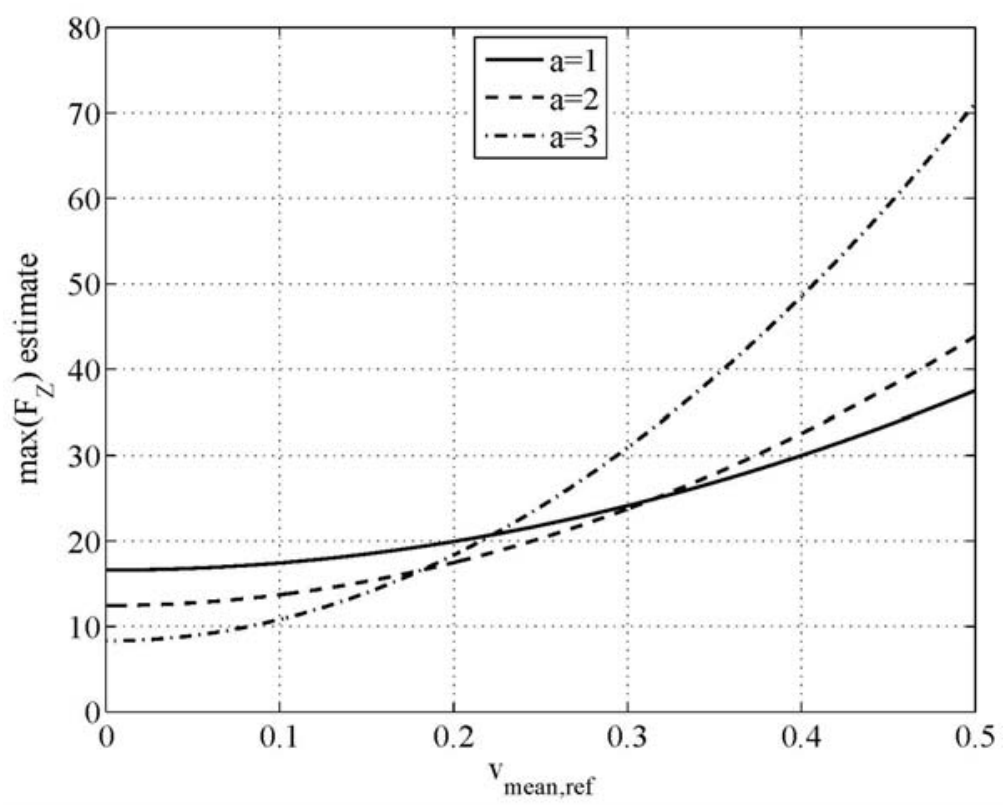

Figure 10. System with $N=4$; estimate of the maximum spike force according to (14) vs. $\bar{v}_{\text {ref }}$ according to (4.15), shown for different numbers of active spikes; slope $\alpha=25^{\circ}$

Obviously, (4.9) gives the following dependence to convert $f$ and $\bar{v}_{r e f}$ :

$$
f=\frac{\bar{v}_{r e f}}{(N-a) \varepsilon l_{0}} .
$$

As observed in previous simulations, the actual speed resulting from a certain gait frequency can slightly vary, due to the allowed error of $\lambda$. 
The combination of the plotted functions exhibits certain speed intervals:

- For speeds of up to $0.18 \frac{m}{s}$, the gait with $a=3$ requires the lowest spike force maxima;

- $\quad$ Between $0.18 \frac{\mathrm{m}}{\mathrm{s}}$ and $0.31 \frac{\mathrm{m}}{\mathrm{s}}$, the gait with $a=2$ is advantageous;

- For speeds higher than $0.31 \frac{\mathrm{m}}{\mathrm{s}}$, the gait with $a=1$ is superior.

This corresponds qualitatively to the results of the simulations in Section 4.3.1. The exact values of the speed thresholds differ slightly, possibly due to neglecting spring and damping forces.

The relevant parts of this plot are the intersections of the curves. They represent the speed thresholds at which the number of active spikes should be changed to achieve the lowest possible spike forces. The speed thresholds are noted as $\bar{v}_{r e f, q \leftrightarrow q+1}($ with $q=1, \ldots, n-1$ ): meaning the gait reference speed at which the number of active spikes should be switched from $q$ to $q+1$ or $q+1$ to $q$.

The switching rule is the following:

- $\quad$ For speeds smaller than $\bar{v}_{r e f, q \leftrightarrow q+1}$, switch to $a=q+1$ (larger number of active spikes);

- $\quad$ For speeds larger than $\bar{v}_{r e f, q \leftrightarrow q+1}$, switch to $a=q$ (smaller number of active spikes).

To find these thresholds, (14) is used to find the frequency, and (9) is used to get the corresponding speed, for the case $N=4$.

Speed threshold 1:

$$
\begin{gathered}
F_{Z, \max }\left(f=f_{1}, a=1\right)=F_{Z, \max }\left(f=f_{2}=\frac{3}{2} f_{1}, a=2\right) \Rightarrow f_{1}=\sqrt{\frac{g \sin (\alpha)}{3 N^{2} \pi \varepsilon l_{0}}} \\
\Rightarrow \bar{v}_{r e f, 1 \leftrightarrow 2}=\left(1-\frac{1}{N}\right) \sqrt{\frac{1}{3 \pi} \varepsilon l_{0} g \sin (\alpha)}
\end{gathered}
$$

Speed threshold 2:

$$
\begin{gathered}
F_{Z, \max }\left(f=f_{2}, a=2\right)=F_{Z, \max }\left(f=f_{3}=2 f_{2}, a=3\right) \Rightarrow f_{2}=\sqrt{\frac{g \sin (\alpha)}{4 N^{2} \pi \varepsilon l_{0}}} \\
\Rightarrow \bar{v}_{\text {ref }, 2 \leftrightarrow 3}=\left(1-\frac{2}{N}\right) \sqrt{\frac{1}{4 \pi} \varepsilon l_{0} g \sin (\alpha)}
\end{gathered}
$$

Summarizing, the general form of these speed thresholds is, for arbitrary $\mathrm{N}$ (number of mass points),

$$
\bar{v}_{r e f, q \leftrightarrow q+1}=\left(1-\frac{q}{N}\right) \sqrt{\frac{1}{2 \pi}\left(1-\frac{1}{N-q}\right) \varepsilon l_{0} g \sin (\alpha)},
$$

whereby these speed thresholds only depend on $N$ and $\alpha$, see [10].

Remark 4.3: Note, that we only assumed that all masses are equal, i.e., $m_{i}=m$, their actual value is not required to find these speed thresholds for switching the number of active spikes. 
If the slope is known (measured), it is then possible to choose adequate gaits in a meaningful way, with respect to low spike forces. The estimate of the actuator forces, see (11), gives identical speed thresholds, as it differs from the spike force estimate only by the addition of a weight term of one mass; this term does not influence the intersection of curves for different $a$. It should therefore be possible to use the speed thresholds above to achieve optimal locomotion, i.e., to use the gait that yields the lowest actuator/spike forces.

\subsubsection{Proportional frequency control}

It appears useful to apply closed-loop control between the input variable reference frequency and the output variable, which may be the measured (maximum) value of actuator forces, spike forces or actuator powers. The implementation is based on simple P-feedback. First, a reference value $X_{\text {ref }}$ for the output criterion is chosen. The actual output is built by measurements of the chosen criterion over given intervals of time, in this case $\frac{T}{N}$. Measurement and frequency adjustment according to the control are evaluated after these $\frac{T}{N}$ time intervals, so that the result is a time-discrete controller which provides discrete frequency values $f_{k}=f\left(k_{\bar{N}}^{T}\right)$.

The deviation between the measurement variable and its reference is evaluated and fed back into the loop to form the new frequency. The measurement shall be performed continuously over given time intervals, in order to find the maximum value, i.e.,

$$
X_{\text {err }, k}:=X_{\text {ref }}-X_{\text {meas }, k} \text {, with } X_{\text {meas }, k}=\max _{\tau \in\left[\frac{T}{4}(k-1), \frac{T}{4} k\right]}\left\{\left|X_{0}(\tau)\right|, \ldots,\left|X_{n}(\tau)\right|\right\} .
$$

This error shall contribute to the factor of frequency adjustment proportionally. For the frequency adjustment, a factor is used instead of absolute values, because a feasible range of frequencies might not be known in advance and a relative factor should enable proper adjustment for any range. The new frequency is calculated according to

$$
f_{k+1}=f_{k}+f_{k} X_{e r r, k} k_{P}=f_{k}\left(1+X_{e r r, k} k_{p}\right) \text {, }
$$

with a gain $k_{P} \in \mathbb{R}_{+}, f_{0} \in \mathbb{R}_{+}$of the frequency P-feedback. As described before, this implementation entails relative frequency changes, where a certain deviation of the measured variable from its reference always results in the same percental frequency change. For practical implementations, the variable $X$ can represent actuator forces, actuator powers, or spike forces.

\subsubsection{Simulations}

In the following simulation for the WLLS with $N=4$, the parameter set from Table 5 is used. The slope is defined as a function of time:

$$
\alpha(t)=\left\{\begin{array}{lc}
15^{\circ}, & t \leq 10 s \\
60^{\circ}, & 10 s<t \leq 20 s \\
25^{\circ}, & t \geq 20 s
\end{array}\right.
$$

Three gaits for different numbers of active spikes are chosen from the set of optimal gaits found in Section 3.4:

- $\boldsymbol{A}_{0}=\{1\}, \operatorname{dir}=l$

- $\boldsymbol{A}_{0}=\{1,2\}, \operatorname{dir}=l$

- $\boldsymbol{A}_{0}=\{2,3,0\}, \operatorname{dir}=l$

As an initial value, the gait with $a=1$ and a frequency of $\left.f\right|_{t=0}=0.5 \mathrm{~Hz}$ is selected. The frequency is adjusted by the frequency P-feedback (20) presented before.

The frequency thresholds for the shift of the number of active spikes are derived according to (16) and (17). They are 


$$
f_{1}(\alpha)=\sqrt{\frac{g \sin (\alpha)}{3 N^{2} \pi \varepsilon l_{0}}} \approx 0.4032 \sqrt{\sin (\alpha)} H z \text { and } f_{2}(\alpha)=\sqrt{\frac{g \sin (\alpha)}{4 N^{2} \pi \varepsilon l_{0}}} \approx 0.3492 \sqrt{\sin (\alpha)} H z
$$

With these thresholds, the rule for switching the number of active spikes is

$$
\left\{\begin{array}{cc}
\text { if } a=2 \wedge f \geq \frac{3}{2} f_{1}: & \text { switch to } a=1 \\
\text { if }\left(a=1 \wedge f<f_{1}\right) \vee\left(a=3 \wedge f>2 f_{2}\right): & \text { switch to } a=2 \\
\text { if } a=2 \wedge f<f_{2}: & \text { switch to } a=3 \\
\text { else: } & \text { no switch }
\end{array}\right\}
$$

When switching $a$, a frequency change is applied to keep the same reference speed as before. So, due to (9), when switching

- From $a=1$ to $a=2$, the frequency is multiplied by $\frac{3}{2}$,

- From $a=2$ to $a=1$, the frequency is multiplied by $\frac{2}{3}$,

- From $a=2$ to $a=3$, the frequency is multiplied by 2 ,

- From $a=3$ to $a=2$, the frequency is multiplied by $\frac{1}{2}$.

\section{Example 4.4: Automatic shifting with spike forces as a criterion}

For the frequency P-feedback according to (19) and (20), the following parameters are chosen:

$$
F_{Z, r e f}=20 \mathrm{~N} \text { and } k_{P}=0.03 \frac{1}{N} .
$$

The gain $k_{P}$ is roughly estimated with a rather small value which means that, e.g., a measured spike force maximum of $30 \mathrm{~N}$ results in a frequency reduction of $30 \%$. With a very small gain $k_{P}$, the adjustment would require many steps; a large value could lead to an oscillating frequency around a stationary value.

In the following figures, the points of slope change (at $10 \mathrm{~s}$ and at $20 \mathrm{~s}$ ) are marked by red lines; blue lines denote points of gait shift.

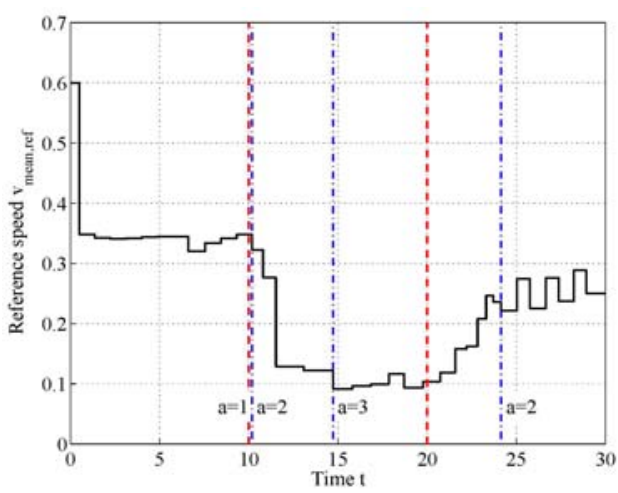

Reference speed $\bar{v}_{r e f}$
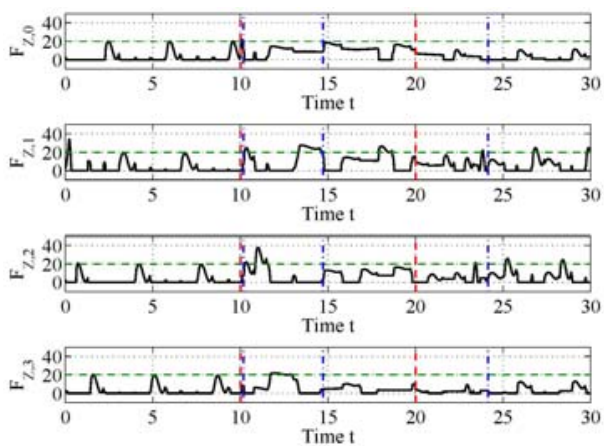

Spike forces

Figure 11. Spike force dependent shifting of gait frequency and number of active spikes, Plots a).

In result, for the larger slope, the gait is almost instantly shifted to $a=2$ (see Figure 11, left). This happens even before the reference speed is reduced, because the (measured) larger slope influences $\bar{v}_{1 \rightarrow 2, N=4}$, as seen in (16), so that the gait with 
$a=2$ is considered optimal. Within about $5 s$ after the slope increase, the speed is reduced to a value that requires the gait with $a=3$ to be used. Later, when the slope is reduced, the shifting back to $a=2$ is done within $4 s$.

Within $t \in[0,10 s]$ the maxima of the spike forces (see Figure 11, right) adhere to the reference value very closely. Later, when different gaits are used, the maxima of some of the spike forces clearly exceed the reference value once per period by up to $30 \%$. If there are strict limits of the maximum allowed spike forces, this deviation should be considered and avoided, e.g. by measuring the values over a full period and only then allow the frequency to be increased.

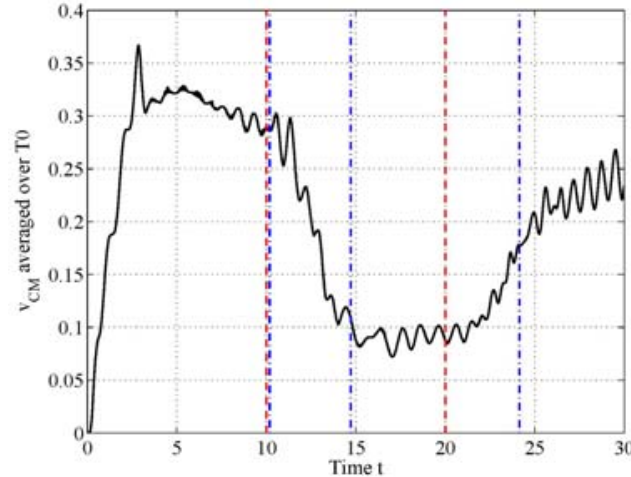

Moving average of locomotion speed

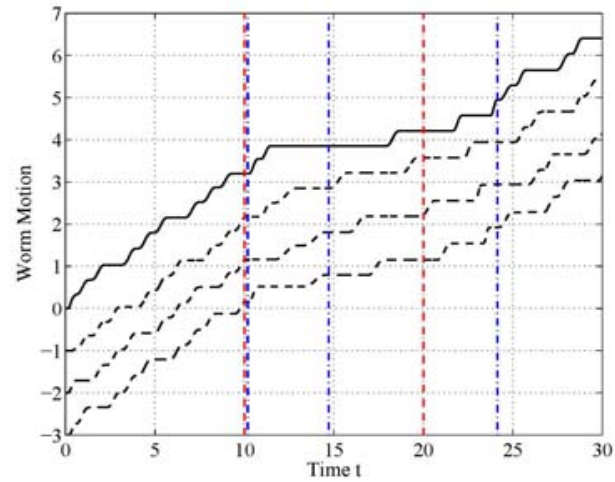

Worm movement

Figure 12. Spike force dependent shifting of gait frequency and number of active spikes, Plots b).

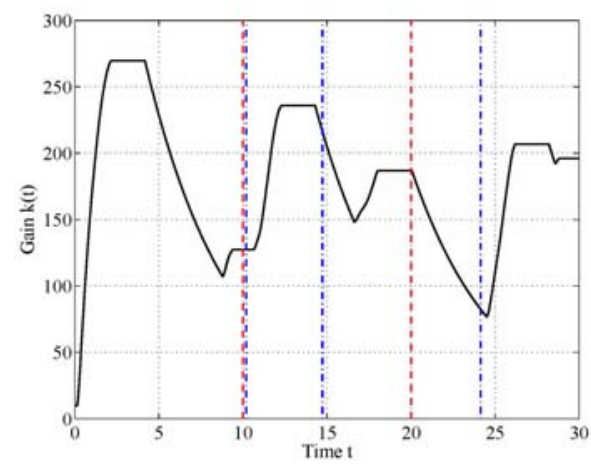

Gain $k(t)$

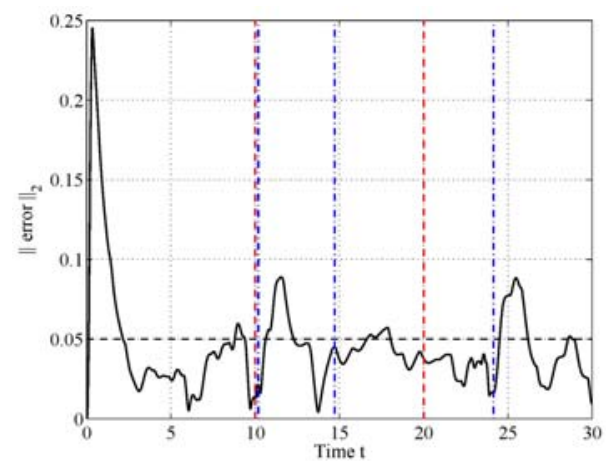

Error norm

Figure 13. Spike force dependent shifting of gait frequency and number of active spikes, Plots c).

In the plot of the worm motion (Figure 12, right), the different movement patterns of the gaits are visible. Observing $x_{0}$ for $t \in[11 \mathrm{~s}, 18 \mathrm{~s}]$, a remarkably long phase in which the front element of the WLLS rests can be seen. It is the result of a certain combination of different gaits, $a=2$ and $a=3$, which has this one mass point rest for $5 \frac{T}{4}$ while the gait patterns dictate movement for the other three mass points.

The error norm (see Figure 13, right) is found to reach relatively large values right after each of the gait shifts. At the shift from $a=1$ to $a=2$, it is predominantly the larger slope that requires a higher gain $k($.$) (see Figure 13, left). At the last$ shift, from $a=3$ back to $a=2$, the error norm increases beyond $\lambda$ again, because the new gait has higher requirements with respect to the gain (as can be seen in the gait analysis in Section 3.4). Both of these oversteppings of the error norm last for about $1.7 \mathrm{~s}$. 


\section{Example 4.5: Automatic shifting with actuator forces as a criterion}

The parameters of the frequency controller are given as

$$
u_{\text {ref }}=20 N \text { and } k_{P}=0.03 \frac{1}{N^{*}}
$$

Figures 14 to 16 show the results of the simulation, which are mostly similar to the previous example.

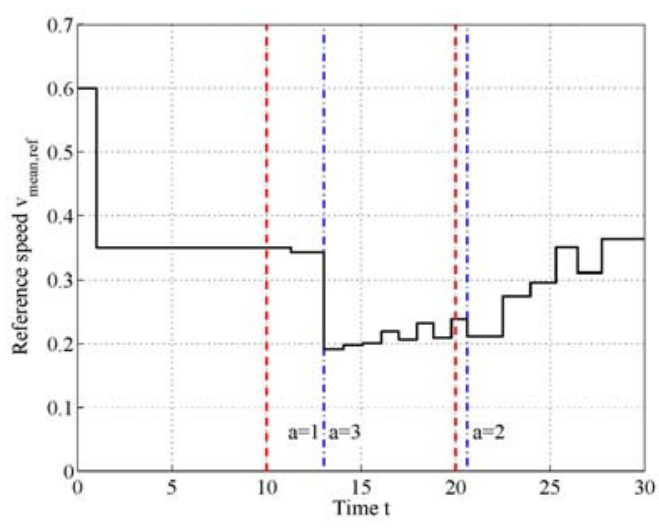

Reference speed $\bar{v}_{r e f}$
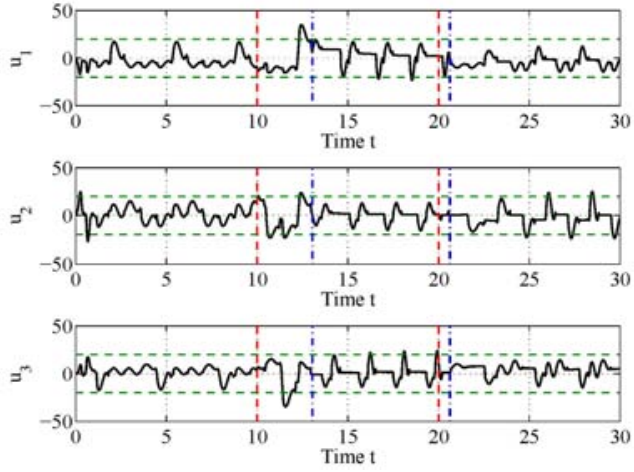

Actuator forces

Figure 14. Actuator force dependent shifting of gait frequency and number of active spikes, Plots a).

Right at the beginning, the reference speed (see Figure 14, left) is reduced, since the upper speed limit for forward skidding (12) is already exceeded here. $\bar{v}_{r e f}$ is reduced to the maximum allowed speed, so that the actuator forces stay well below their reference value. Shortly after the slope increase $(t=10 \mathrm{~s})$, the actuator forces are marginally larger than their reference value, which results in very small steps of frequency decrease. As larger deviations from the actuator force reference occur, the reference speed is reduced further and the speed threshold for switching to the gait with $a=3$ is reached. The speed interval for $a=2$ is skipped here. In the last section of an $\alpha=25^{\circ}$-slope, the gait is switched to $a=2$ even before the speed is increased, because of the influence of the reduced slope $\alpha$ on the value of the speed threshold, as defined in (17).

The (absolute) actuator force extremes (see Figure 14, right) are essentially (with small deviations) brought to the reference value by the frequency controller.

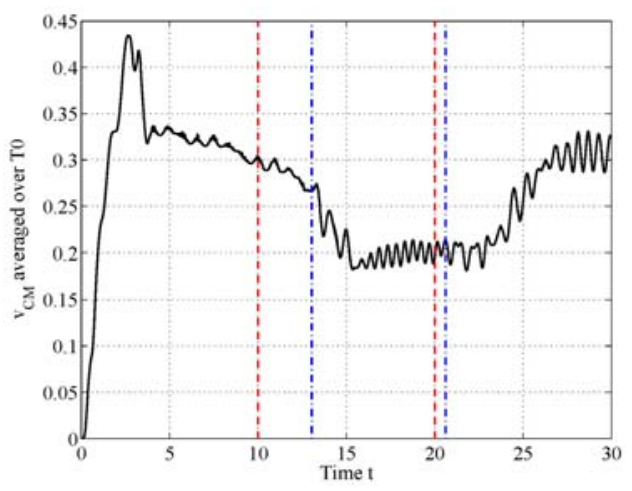

Moving average of locomotion speed

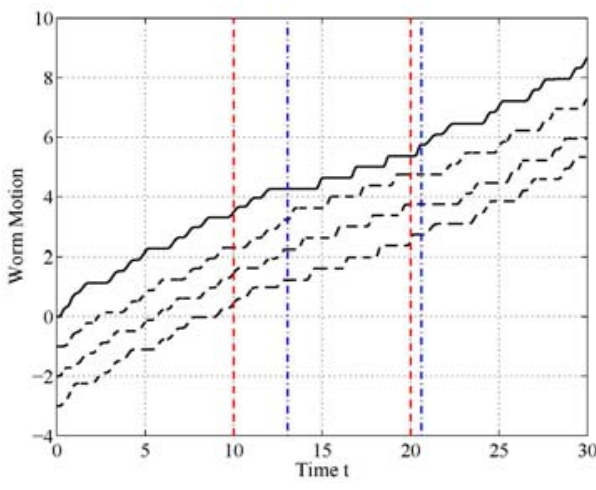

Worm movement

Figure 15. Actuator force dependent shifting of gait frequency and number of active spikes, Plots b). 


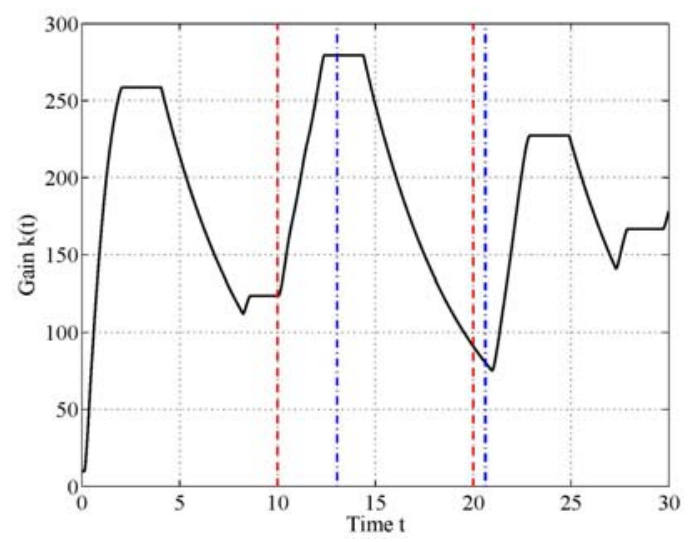

Gain $k(t)$

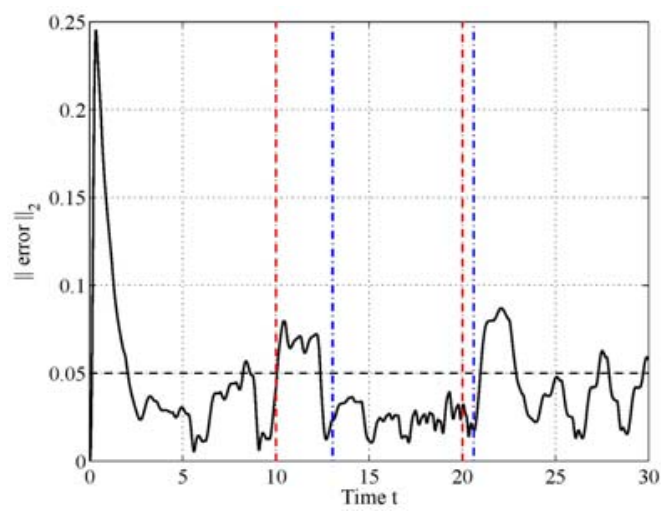

Error norm

Figure 16. Actuator force dependent shifting of gait frequency and number of active spikes, Plots c).

\section{Conclusions \& Outlook}

The advantage of adaptive control for the dynamical realization of the peristaltic motion of artificial worm systems is shown. The (adaptive) control was directed, based on the dynamical motion equations, to ensure a prescribed gait (i.e., a temporal pattern of shape - something internal!) in order to track desired movements from kinematical theory. The foregoing considerations have shown that adaptive control is promising in application to worm-like robotic locomotion systems, where we applied an already existing and best working adaptive controller. Optimal kinematic gaits can be determined in using a gait generation algorithm incorporating the dynamics of the system. These optimal kinematic gaits lead to the basic idea of shifting of gaits in a variable environment (i.e., changing slope). Overall, the adaptive controllers are capable of quickly adjusting the gain when the frequency of the gait and/or the gait pattern are changed, such that the control goal can be accomplished. The combined switching of frequency and number of active spikes enables quick speed adjustments in order to adhere to given limits of a system variable such as spike or actuator force - although these limits may be exceeded for short amounts of time, because this is a necessary trigger for the speed control. The gait shift, i.e., the change of the number of active spikes, helps to choose the gait that is optimal for a certain speed and slope. With this, predefined restrictions of forces or powers of the system are utilized in such a way that the maximum speed is reached. Future work shall be directed to improvements of the concepts for automatic gait shift, in order to achieve faster and more robust shifting; implementations of these shifting concepts for WLLS with a larger number of segments, $N>4$; experimental verification of spike force estimations, speed intervals and gait shift.

\section{References}

[1] Behn, C.; Zimmermann, K.: Adaptive $\lambda$-tracking for locomotion systems; in: Robotics and Autonomous Systems. 2006; 54(7): 529-545. http://dx.doi.org/10.1016/j.robot.2006.04.005

[2] Behn, C.: Adaptive Control of Straight Worms without Derivative Measurement; in: Multibody System Dynamics. 2011; 26(3): 213-243. http://dx.doi.org/10.1007/s11044-011-9250-2

[3] Behn, C.; Steigenberger, J.: Experiments in Adaptive Control of Uncertain Mechanical Systems; in: International Review of Mechanical Engineering. 2010; 4(7).

[4] Behn, C.; Steigenberger, J.: Adaptively controlled bio-inspired locomotion systems with friction; 11th Conference on Dynamical Systems - Theory and Applications; Łódź, Poland, 2011.

[5] Chen, I.-M.; Yeo, S.H.; Gao, Y.: Gait generation for inchworm-like robot locomotion using finite state mode; in: Proceedings 1999 IEEE International Conference on Robotics and Automation. 1999; 3: 2026-2031. 
[6] Chen, I.-M.; Yeo, S.H.; Gao, Y.: Locomotive gait generation for inchworm-like robots using finite state approach; in: Robotica. 2001; 19: 535-542. http://dx.doi.org/10.1017/S0263574700003271

[7] Ilchmann, A.: Non-Identifier-Based High-Gain Adaptive Control; Springer, London, 1993.

[8] Loepelmann, P.: Design and Analysis of Control Strategies for biologically inspired Sensor Systems; Master Thesis, TU Ilmenau (Germany), 2011.

[9] Ostrowski, J.; Burdick, J.; Lewis, A.D.; Murray, R.M. The Mechanics of Undulatory Locomotion: The Mixed Kinematic and Dynamic Case; in: Proceedings IEEE International Conference on Robotics and Automation, 1995.

[10] Schwebke, S.: Contributions to adaptive control strategies of biomimetic, worm-like locomotion systems and their use for gait shifting; Bachelor Thesis, TU Ilmenau (Germany), 2012.

[11] Seok, S.; Onal, C.D.; Wood, R.; Rus, D.; Kim, S.: Peristaltic locomotion with antagonistic actuators in soft robotics; in: Proceedings IEEE International Conference on Robotics and Automation, May 2010, Anchorage (Alaska).

[12] Slatkin, A.B.; Burdick, J.; Grundfest, W.: The development of a robotic endoscope; in: Proceedings 1995 IEEE/RSJ International Conference on Intelligent Robots and Systems 95 - Human Robot Interaction and Cooperative Robots. 1995; 2: $162-171$.

[13] Steigenberger, J.; Behn, C.: Gait generation considering dynamics for artificial segmented worms; in: Robotics and Autonomous Systems. 2011; 59: 555-562. http://dx.doi.org/10.1016/j.robot.2011.04.002

[14] Steigenberger, J.; Behn, C.: Worm-like locomotion systems: an intermediate theoretical approach; Oldenbourg, Munich, 2012. 